approximation [5] is obtained by neglecting differences in $\alpha$ altogether in (19). The iteration

$$
\psi_{n+1}=g^{-1} \int_{\theta_{N}}^{\theta}(g / D)\left[h+k \int_{0}^{\psi_{n}}\left(\alpha_{s}-\alpha\left(\psi^{\prime}\right)\right) d \psi^{\prime}\right] d \theta, \quad n \geq 1,
$$

converges when $\theta / \theta_{N}$ is bounded, and the solution is $\psi_{\cdot}(\theta)=\psi_{2}(\theta)\left[1+0\left(\alpha_{m}^{2}\right)\right]$, where $\alpha_{m}$ denotes the maximum difference in $\alpha$ occurring between the body and the streamline with direction $\theta$ at the shock. The shape of the shock is found from (12) by quadrature. The distributions of $S$ and $M$ in the characteristic plane are also determined by $\psi_{s}(\theta)$, since $S$ and $\alpha$ are known functions of $\theta$ at the shock, and the distributions in the flow plane are again found from (12).

When the region of flow is unlimited, $l / D \sim 2 / \theta$ as $\theta \rightarrow 0$, and hence $g \sim \theta^{2}$; but if $\alpha_{1}$ denotes the value of $\alpha$ upstream of the shock, then [6] $\alpha_{1}-\alpha_{a}(\theta)=0\left(\theta^{3}\right)$ if $\theta$ is sufficiently small throughout the flow, and since [5] $\psi_{\mathrm{a}} \sim \theta^{-2}$, the iteration still converges and the solution is again $\psi_{1}=\psi_{2}\left[1+O\left(\alpha_{m}^{2}\right)\right]$.

It may be noted that the theory applies also to the flow downstream of several shocks facing in the same direction, but since $D$ depends not only on $\theta$, when the flow upstream of the shock is non-uniform, the determination of the second and further shocks is more laborious.

\title{
REFERENCES
}

1. J. J. Mahony, A critique of shock-expansion theory, J. Aeronaut. Sci. 22, 673-680 (1955)

2. J. J. Mahony, The flow around a supersonic aerofoil, Aero. Res. Lab. Melbourne Note A 147-1955.

3. P. M. Stocker, Hypersonic flow: part I, Armament Research \& Dev. Establ. (Ft. Halstead) Rep. (B) $22 / 55,1955$.

4. L. Howarth (editor), Modern developments in fluid dynamics: high speed flow, Oxford University Press, 1953.

5. R. Courant and K. O. Friedrichs, Supersonic flow and shock waves, Interscience, N.Y., 1948.

6. R. E. Meyer, Focusing effects in two-dimensional supersonic flow, Phil. Trans. Roy Soc. A242, 153-171 (1949).

\section{ON RICCATI'S RESOLVENT*}

\section{BY AUREL WINTNER (The Johns Hopkins University)}

There belongs to every differential equation of the form

$$
y^{\prime \prime}+f(t) y^{\prime}+g(t) y=0
$$

a "Riccati resolvent,"1 a differential equation which, in contrast to the equation (1) of second order, is not linear (but quadratic in the unknown function) but which is only of the first order. It is also known that a reduction to a differential equation of Riccati type remains possible if the single equation (1) of second order is generalized to a system of $n=2$ equations of first order, that is, to

$$
u^{\prime}=a(t) u+b(t) v, \quad v^{\prime}=a(t) u+d(t) v
$$

*Received February 29, 1956.

'See, e.g., F. Klein, Vorlesungen ueber die hypergeometrische Funktion, Berlin, 1933, pp. 150-154. 
$\left(u=y, v=y^{\prime}\right)$. But if $n=2$ is replaced by an arbitrary $n$, then, since the usual transition from the two equations (2) to a Riccati equation is based on a highly explicit calculation (see Eqs. (11), (12) below), it does not seem to have been noted in the literature that Riccati's equation (for $n=2$ ) has a generalization (for any $n$ ). This generalization, suggested by the formal separation used for quite another (in fact, asymptotic) purpose in an earlier note ${ }^{2}$, explains, among other things, the explicit simplifications taking place in the classical case $n=2$, a case which thus turns out to be of misleading simplicity.

Let $A$ be an $n$ by $n$ matrix, given as a continuous function $A(t)$ on a $t$-interval, and let the system (2), where $n=2$, be generalized to

$$
x^{\prime}=A(t) x,
$$

where $x$ is a vector with $n$ components (which are $u$ and $v$ if $n=2$ ). In order to simplify the notations, suppose that all $n^{2}$ elements of $A$ are real-valued for all $t$ (actually, this involves no loss of generality, since $n$ can be replaced by $2 n$ ). Then, if $x(t)$ is any solution vector, its components can be assumed to be real. If $x(t)=0$ holds for a single $t=t_{0}$, then it holds for every $t$, since $x(t) \equiv 0$ is one, hence the only, solution of (3) satisfying the initial condition $x\left(t_{0}\right)=0$. Thus, if this trivial solution of (3) is disregarded, it can be assumed that $r>0$ holds for all $t$, where $r=r(t)$ denotes the length $|x|=|x(t)|$ of the vector. Hence $x(t)$ can be written as $r(t) e(t)$, where $e(t)$ is a unique vector of unit length.

Since the unknown vector function $e=e(t)$ has $n$ components $e_{1}, \cdots, e_{n}$ subject to the condition $e \cdot e=1$, its determination requires that of $n-1$ scalar functions. On the other hand, it turns out that (i) the "phase vector" $e(t)$ of

$$
x(t)=r(t) e(t), \text { where }|e|=1, \quad r=|x| \neq 0,
$$

has a differential equation which does not contain the length $r(t)$ of $x(t)$ and that (ii) if a solution $e(t)$ of that differential equation is known, then a corresponding scalar $r(t)$, one for which the product (4) becomes a solution of the given differential equation (3), can be obtained by a quadrature.

First, substitution of $x=r e$ into the vector equation (3) shows that $r^{\prime} e+r e^{\prime}=$ $r A(t) e$ or, since $r>0$,

$$
(\log r)^{\prime} e+e^{\prime}=A(t) e .
$$

Hence, scalar multiplication by $e$ leads to

$$
(\log r)^{\prime}=e \cdot A(t) e,
$$

since $e \cdot e \equiv 1$ implies that $e \cdot e^{\prime} \equiv 0$. But the two equations containing $(\log r)^{\prime}$ show that

$$
e^{\prime}=\{A(t)-[e \cdot A(t) e] I\} e,
$$

where $I$ denotes the unit matrix. Clearly, the (non-linear) differential equation (6) is of the type claimed under (i), and the quadrature referred to under (ii) is that assigned by the relation (5).

The system (6) for the components $e_{i}$ of the phase vector $e=\left(e_{1}, \cdots, e_{n}\right)$ is a system of order $n$ but can be replaced by a system of order $n-1$ (for $e_{1}, \cdots, e_{n-1}$ only), since

$$
e_{n}=\left(1-e_{1}^{2}-\cdots-e_{n-1}^{2}\right)^{1 / 2} .
$$

${ }^{2}$ A. Wintner, Quart. Appl. Math. 8, 102-104 (1950). 
But it remains to be ascertained that the condition $|e(t)|=1$ is satisfied for all $t$ if it is satisfied for a single $t$. In fact, thus far it was a tacit assumption that if $e(t)$ is any solution of (6) which belongs to an arbitrary initial condition $e\left(t_{0}\right)$ satisfying $\left|e\left(t_{0}\right)\right|=1$, then $\mid e(t) !=1$ will hold for all $t$. This can however be verified as follows:

Scalar multiplication of the equation (6) by the vector $e$ gives

$$
e \cdot e^{\prime}=e \cdot A(t) e-e \cdot[e \cdot A(t) e] e,
$$

since $I e=e$. In view of $2 e \cdot e^{\prime}=\left(e^{2}\right)^{\prime}$, where $e^{2}=e \cdot e=|e|^{2}$, this can be written in the form

$$
\left(1-|e|^{2}\right)^{\prime}=-2[e \cdot A(t) e]\left(1-|e|^{2}\right) .
$$

Accordingly, if $\epsilon=\epsilon(t)$ is an abbreviation for $1-|e(t)|^{2}$ and if $\mu$ denotes the maximum of $: e(t) \cdot A(t) e(t) \mid$ on a $t$-interval on which a solution $e(t)$ of $(6)$ is given, then

$$
\left|\epsilon^{\prime}(t)\right| \leqq 2 \mu|\epsilon(t)|
$$

holds on that $t$-interval. Let $t_{0}$ and $t>t_{0}$ be in the latter. Then, since $\mu$ is a constant, it can readily be concluded from the differential inequality (8) that the absolute value of any $\epsilon(t)$ is majorized by that solution $E(t)$ of the differential equation $E^{\prime}=2 \mu E$ which belongs to the initial condition $E\left(t_{0}\right)$ having the value $\left|\epsilon\left(t_{0}\right)\right|$. Consequently,

$$
|\epsilon(t)| \leqq \epsilon\left(t_{0}\right) \exp \left\{2 \mu\left(t-t_{0}\right)\right\},
$$

since the product on the right is identical with $E(t)$. It follows that $\epsilon(t)=0$ holds for every $t$ if it holds for $t=t_{0}$. Since $\epsilon$ was an abbreviation for $1-|e|^{2}$, this proves that $|e(t)|=1$ holds for every $t$ if it holds for $t=t_{0}$.

In order to see that the rule consisting of (ii) and (i) reduces to the use of Riccati's resolvent in the classical case, let $n=2$. Then the vectorial differential equation (3) becomes the binary system (2), with

$$
x=\left(\begin{array}{l}
u \\
v
\end{array}\right), \quad A(t)=\left(\begin{array}{ll}
a(t) & b(t) \\
c(t) & d(t)
\end{array}\right) .
$$

Correspondingly, the "polar factorization" (4) reduces to the introduction

$$
u(t)=r(t) \cos \varphi(t), \quad v(t)=r(t) \sin \varphi(t) \quad(r>0)
$$

of polar coordinates in the $(u, v)$-plane, since $|e|=1$ or (7) now becomes

$$
e=\left(\begin{array}{l}
e_{1} \\
e_{2}
\end{array}\right), \quad e_{1}=\cos \varphi, \quad e_{2}=\sin \varphi
$$

for a suitable $\varphi=\varphi(t)$ [which, by continuity, is uniquely determined by an initial $\varphi\left(t_{0}\right)$; the latter constant is determined $\bmod 2 \pi$ if $t_{0}$ and the solution $u=u(t), v=v(t)$ of (2) are given]. But direct substitutions show that the relations (9), (10) reduce the scalar equation (5) to

$$
(\log r)^{\prime}=a(t) \cos ^{2} \varphi+[b(t)+c(t)] \cos \varphi \sin \varphi+d(t) \sin ^{2} \varphi
$$


and the $n$ scalar equations, represented by vector equation (6), to $n=2$ differential equations for the functions $\cos \varphi, \sin \varphi$, which $n=2$ differential equations are, however, equivalent to the $n-1=1$ differential equation

$$
\varphi^{\prime}=c(t) \cos ^{2} \varphi+[d(t)-a(t)] \cos \varphi \sin \varphi-b(t) \sin ^{2} \varphi .
$$

This is the classical result ${ }^{3}$, since (12) can be written in the form ${ }^{4}$

$$
(\tan \varphi)^{\prime}=c(t)+[d(t)-a(t)] \tan \varphi-b(t) \tan ^{2} \varphi,
$$

which is a Riccati equation for $\tan \varphi$.

If $n=3$, then the identity (7) shows that the substitution (10) can be replaced by

$$
e_{1}=\cos \varphi \cos \theta, \quad e_{2}=\cos \varphi \sin \theta, \quad e_{3}=\sin \varphi
$$

and (6), instead of being a single non-linear differential equation (12), becomes a system of $n-1=2$ (non-linear) differential equations for $\varphi=\varphi(t)$ and $\theta=\theta(t)$. It is clear from (7) how (13) can be extended to any $n$.

There is a trivial case for every $n$, the case in which the coefficient matrix $A(t)$ of the system (3) is skew-symmetric (for every $t$ ). In fact, a bilinear form $z \cdot A x$ belonging to a skew-symmetric matrix $A$ vanishes whenever $z=x$. Hence (6) becomes identical with the case $x=e$ of (3), and (5) reduces to $(\log r)^{\prime}=0$ or $r(t)=$ constant, that is, to the well-known fact that $|x(t)|^{2}=$ constant in a first integral of (3) whenever the coefficient matrix $A(t)$ is skew-symmetric for every $t$.

3See T. Levi-Civita, Ann Ec. Norm. Sup. ser. 3, 28, 352-353 (1911); A. Wintner, The analytica foundations of celestial mechanics, Princeton University Press, 1941, p. 379.

In order to avoid the infinities of $\tan \varphi$, Levi-Civita (loc. cit.) replaces the (real) Riccati equation (12 bis) by a (complex) Riccati equation for exp $2 i \varphi$. 